\title{
Fencing artificial waterpoints failed to influence density and distribution of red kangaroos (Macropus rufus)
}

\author{
Y. Fukuda ${ }^{\mathrm{A}, \mathrm{B}}$, H. I. McCallum ${ }^{\mathrm{A}}$, G. C. Grigg ${ }^{\mathrm{A}}$ and A. R. Pople ${ }^{\mathrm{A}}$ \\ ${ }^{A}$ School of Biological Sciences, The University of Queensland, Brisbane, Qld 4072, Australia. \\ ${ }^{\mathrm{B}}$ Corresponding author. Email: yuki_fukuda@hotmail.com
}

\begin{abstract}
Provision of artificial waterpoints in Australian rangelands has resulted in an increase in the range and density of kangaroos. At high densities, kangaroos can inhibit vegetation regeneration, particularly in some protected areas where harvesting is prohibited. Fencing off waterpoints has been proposed to limit these impacts. Our aim was to determine whether fencing off waterpoints during a drought (when kangaroos would be especially water-limited) would influence the density and distribution of red kangaroos (Macropus rufus). Two waterpoints were fenced within the first 6 months of the 27-month study and a further two waterpoints were kept unfenced as controls in Idalia National Park, western Queensland. We estimated kangaroo densities around waterpoints from walked line-transect counts, and their grazing distribution from dung-pellet counts. Fencing off waterpoints failed to influence either the density or distribution up to $4 \mathrm{~km}$ from the waterpoints. Our results indicate that food availability, rather than the location of waterpoints, determines kangaroo distribution. Few areas in the rangelands are beyond kangaroos' convenient reach from permanent waterpoints. Therefore, fencing off waterpoints without explicitly considering the spatial context in relation to other available water sources will fail to achieve vegetation regeneration.
\end{abstract}

\section{Introduction}

Water is one of the major factors limiting the density and distribution of large herbivores in the arid regions of the world (Western 1975). Consequently, artificial waterpoints are frequently provided to distribute the grazing pressure and increase the carrying capacity of arid and semiarid areas for domestic and wild herbivores. Accumulating evidence shows that provision of artificial waterpoints has had a negative and often unintended impact on biodiversity (de Leeuw et al. 2001; Smit et al. 2007) and, although the issues remain controversial in the USA (Rosenstock et al. 1999), the need to develop appropriate surface-water management policies that maximise biodiversity conservation is becoming realised (OwenSmith 1996; Harrington et al. 1999). In the Australian rangelands, European settlers have provided large numbers of artificial waterpoints to support livestock. Today, little land remains beyond $2-3 \mathrm{~km}$ from waterpoints in sheep country, and $6 \mathrm{~km}$ in areas where cattle are grazed (James et al. 1999). The increased availability of water has had an unintended impact on large, native herbivores, particularly kangaroos, leading to an increase in their range and density (Calaby and Grigg 1989; Pople 2006). At high densities, kangaroos often inhibit vegetation regeneration (see Grice and Barchia 1992; Norbury et al. 1993; Sluiter et al. 1997; Pople and McLeod 2000). This severely compromises conservation goals in some protected areas (where commercial harvesting is prohibited), e.g. by reducing habitats and food for threatened fauna (Department of Environment 1998). Since the negative impact of waterpoint provision on biodiversity was reported by Landsberg et al. (1997), some park managers have reacted by initiating programs of closing (or fencing off) these artificial waterpoints (e.g. Bennett 1997). The assumption is that this will automatically facilitate regeneration of native plants around the waterpoints because kangaroos will move closer to other water sources, reducing the local densities. This concerns surrounding land owners because they fear that kangaroos will be forced out on to their properties where they will then compete with livestock for forage (Pople and Page 2001).

The density of kangaroos, especially when conditions are mild, is limited primarily by forage abundance (Bayliss 1987). Under mild conditions, water may not be an important limiting factor, and removing kangaroos' access to water may have little effect on their densities. This was illustrated by Montague-Drake (2004) in Sturt National Park where closing two artificial waterpoints for up to 4 months had no effect on the density of kangaroos. However, water may become an important limiting factor when conditions get hotter and drier because drinking requirements of kangaroos increase substantially (McCarron and Dawson 1989). Without sufficient water to aid digestion, malnutrition can lead to death. This suggests that, especially in dry conditions, kangaroos will abandon inaccessible waterpoints and move closer to alternative sources. Previous studies have shown that when conditions deteriorate, and when plants and ephemeral sources provide insufficient water, many large macropods do rely on artificial waterpoints (Ealey 1967; Freudenberger and Hacker 1995; Montague-Drake and Croft 2004). Once their access to artificial water sources is removed, kangaroos move in order to drink from alternative waterpoints within their home range (Norbury 1992; Gibson 1995; Hacker and Freudenberger 1997). This may result in a range shift if the alternative waterpoints are far from the usual feeding grounds and 
reaching them can involve high-energy expenditure. However, no studies have investigated the impact of denying kangaroos an access to water for longer than 4 months and; therefore, only the short-term impact on the density and spatial distribution of kangaroos is known.

Our aim was to determine the effects of fencing off waterpoints on the density of kangaroos over a period of up to 25 months and to compare the spatial distribution of kangaroos between areas with fenced and unfenced waterpoints. The study examined areas up to $4 \mathrm{~km}$ from selected waterpoints so as to be similar to the study by Montague-Drake (2004). Because fencing coincided with a severe drought and alternative waterpoints were available within the kangaroos' home ranges, we expected that once waterpoints were fenced, kangaroo density would decline from the areas surrounding fenced waterpoints. We also expected that the spatial distribution of their grazing would become more focussed around unfenced waterpoints, particularly during dry seasons. If this were the case, it would concur with the philosophy now driving the destruction of many artificial waterpoints in inland national parks.

\section{Materials and methods}

Study area

The study was conducted at Idalia National Park in western Queensland $\left(24^{\circ} 83^{\prime} \mathrm{S}, 144^{\circ} 60^{\prime} \mathrm{E}\right.$; Fig. 1). The semiarid park has a summer-dominated rainfall pattern. The average maximum temperature exceeds $35^{\circ} \mathrm{C}$ during summer. Winter is mild and generally dry. The long-term mean annual rainfall from 1894 to 2004 was $470 \mathrm{~mm}$ (measured on Terrick Terrick, 20 km north-east of the park; B. Gordon, Bureau of Meteorology, pers. comm.). Dissected tableland covers an extensive area of this $1440-\mathrm{km}^{2}$ park and separates pockets of open plains, the preferred habitat of kangaroos. These open plains are dominated by annual grasses, annual forbs, perennial shrubs and mixedwoodland species, such as poplar box (Eucalyptus populnea) and brigalow (Acacia harpophylla). The park was gazetted in 1990. Before this, two grazing properties had run cattle and sheep on the land intensively for $\sim 100$ years, particularly in the north-eastern third of the park (Department of Environment 1998). In total, 17 artificial waterpoints (the majority in this area) have remained in operation (Pople and Page 2001).

Two species of large macropods commonly found in the park are the red kangaroo (M. rufus) and the common wallaroo (M. robustus). According to annual aerial surveys by the Queensland Parks and Wildlife Services (QPWS), the average densities (from 1997 to 2001) of red kangaroos and wallaroos in the park were 50.4 and 81.1 individuals per square kilometre, although densities as high as 143.9 and 116.1 individuals per square kilometre, respectively, have been recorded. Idalia National Park plays an important role in recovering the populations of the endangered bridled nailtail wallaby

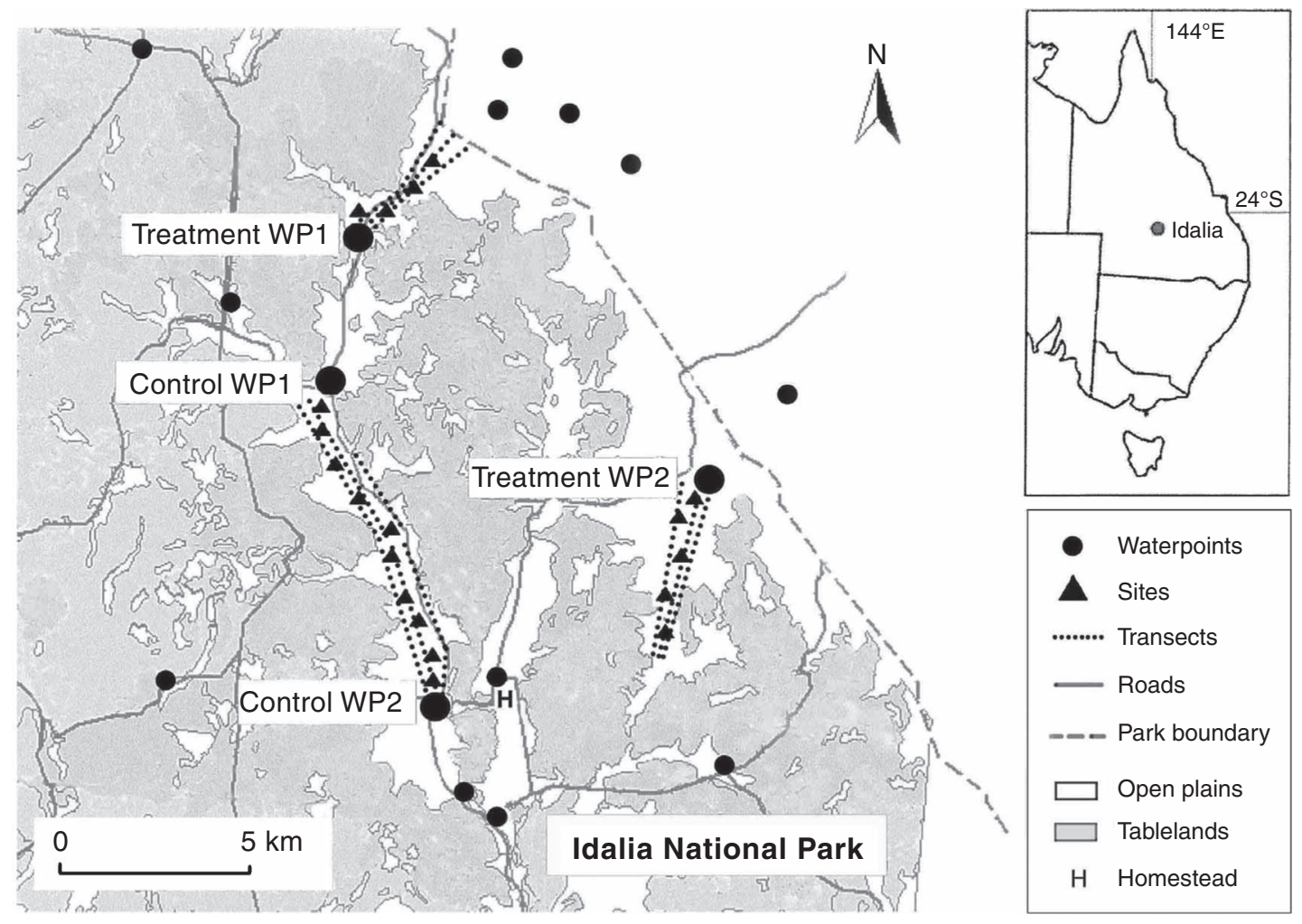

Fig. 1. Location of the four study waterpoints in the north-eastern part of Idalia National Park and other waterpoints near the study area. All, except Control WP1, were artificial waterpoints and held water throughout the study period. Kangaroos also had access to waterpoints on a grazing property adjoining the park. Triangles indicate the location of sites at five distances from each waterpoint at which the grazing pressure and vegetation were monitored. The dotted lines indicate line-transects on which the density kangaroo was monitored. 
(Onychogalea fraenata; Pople et al. 2001). It is considered that large macropods, when occurring at such high densities, increase competition for resources and have a detrimental impact on the wallaby's recovery, although no study has yet been undertaken to determine the extent of their dietary overlap. Other macropod species found in Idalia National Park are the eastern grey kangaroo (M. giganteus, not common), the black striped-wallaby ( $M$. dorsalis), the yellow-footed rock wallaby (Petrogale xanthopus celeris) and the swamp wallaby (Wallabia bicolor, not common). A severe drought in 2002 reduced the densities of red kangaroos and wallaroos to 6.3 and 2.1 individuals per square kilometre, respectively (G. LundieJenkins, QPWS, pers. comm.). The density of red kangaroos recovered during our study period, whereas the density of wallaroos remained too low for any statistical analysis (Fukuda 2006). In the present paper, we focus our attention on red kangaroos.

\section{Experimental design}

Two artificial waterpoints, Hobb's Tank and Chuckster's Bore Dam (hereafter referred to as Treatment WP1 and WP2, respectively; Fig. 1), were fenced with a kangaroo-proof fence in August and December 2002 respectively. Junction Hole and Airstrip Dam (hereafter referred to as Control WP1 and WP2 respectively) remained unfenced throughout our study period. Control WP1 was a natural waterhole whereas Control WP2 was an artificial dam. These control waterpoints were chosen because the surrounding environment (a mixture of open and wooded habitats) matched those surrounding the treatment areas. The areas surrounding Treatment WP1, Treatment WP2, Control WP1 and Control WP2 (where our monitoring was conducted) are referred to as Treatment Area 1, Treatment Area 2, Control Area 1 and Control Area 2, respectively. We created small gaps at the bottom of the fence to allow small mammals (such as the bridled nailtail wallaby) to maintain access to water at treatment waterpoints. Fencing off waterpoints selectively - as opposed to closing waterpoints (where water is no longer available to any wildlife) - removes access to water by certain species. It was, thus, considered more desirable for the park.

Although no published records on the home ranges of red kangaroos in our study areas are available, home ranges of red kangaroos in central western New South Wales are between 11 and $21 \mathrm{~km}^{2}$ (McCullough and McCullough 2000). Kangaroos' home ranges are known to increase during dry periods because they actively search for food and water (Norbury et al. 1994). This means that kangaroos in Treatment Areas 1 and 2 may have drunk from Control WP1 and WP2, respectively, or at waterpoints outside the park (Fig. 1). However, this would incur additional energy costs in terms of travelling to a more remote waterpoint. Rainfall data were also collected at the park homestead.

\section{Estimation of the density of kangaroos}

The density of kangaroos was estimated with walked line-transect counts (Southwell 1994). Three 4-km transects were established in each study area (except for Control Area 1, where one transect was shorter than the other two, owing to a difficult access; Fig. 1). Although the dissected tablelands surrounding the study areas limited the extent to which transects could be arranged randomly, we established all transects within a combination of habitats, including open plain, shrublands and mixed woodlands. The minimum distances walked in one morning between the two transects were $0.23 \mathrm{~km}$ (Treatment Area 1), $0.57 \mathrm{~km}$ (Treatment Area 2) and $0.56 \mathrm{~km}$ (Control Area 1 and 2). Although these distances were small, the extent of bias (because of kangaroos being flushed from one transect to another) is likely to be minimal when, as in our study period, the density of the target population is very low (Southwell 1994).

Starting at dawn, a single observer walked two outside transects in each area and completed the walk within $3 \mathrm{~h}$. The third transect (running between the two outside ones; Fig. 1) was walked on a separate morning. At each sighting, the observer recorded the number of red kangaroos, their distance and bearing to the observer. The distance was determined with a laser rangefinder (Bushnell Yardage Pro-Elite, Bushnell Corporation, Overland Park, KS) and the magnetic bearing with a sighting compass. A hand-held GPS (Garmin GPS 45, Garmin International Inc., Olathe, KS) was used to navigate. Data were collected nine times during a 27-month period (June 2002, October 2002, January 2003, March 2003, June 2003, September 2003, January 2004, April 2004 and September 2004) to monitor closely any changes in densities following the fencing off the waterpoints.

\section{Estimation of the spatial distribution of kangaroo grazing pressure}

We used dung-pellet counts to estimate the spatial distribution of the kangaroo grazing pressure in relation to waterpoint location (Landsberg and Stol 1996). Within each study area, three sites were selected at each of five distances, $0.5,1,2,3$ and $4 \mathrm{~km}$, from the waterpoint (Fig. 1). Our sites were established in open habitats because they had higher forage abundance than elsewhere (Scanlan 2002). The minimum distance between any two sites was $200 \mathrm{~m}$ to facilitate a broader spatial sampling. For Treatment Area 1, because soil types differed at $4 \mathrm{~km}$ from the waterpoint, we selected sites up to $3 \mathrm{~km}$ from the waterpoint only (Fig. 1). Within each site, six fixed circular plots $\left(6.2 \mathrm{~m}^{2}\right)$ were established from which the numbers of dung pellets of red kangaroos were repeatedly counted over time. The dung pellets of red kangaroos were distinguished from those of other macropods, using the field guide by Triggs (1984). Six 1-m fence posts were used to mark the edges of three 70-m vegetation transects, in a triangular formation, and the circular dung plot was established around each fence post (Fig. 2). Pellets were initially cleared from all plots in June 2002. The new pellet numbers were then counted on eight occasions from October 2002 to September 2004, coinciding with the monitoring of kangaroo density (see earlier section).

\section{Vegetation monitoring}

We monitored vegetation within seven $0.25-\mathrm{m}^{2}$ vegetation quadrats spaced at 10-m intervals on each of the 70-m fixed transects (Fig. 2). Seven quadrats in each of the three transects yielded cluster samples of 21 quadrats per site. Within each quadrat, percentage cover (aerial cover rather than basal cover) of perennial grasses was visually estimated $(0 \%, 1 \%, 5 \%$, and then to nearest $10 \%$ ) as was the average height to the nearest $5 \mathrm{~cm}$ (Page 1997). Monitoring was repeated nine times from June 2002 


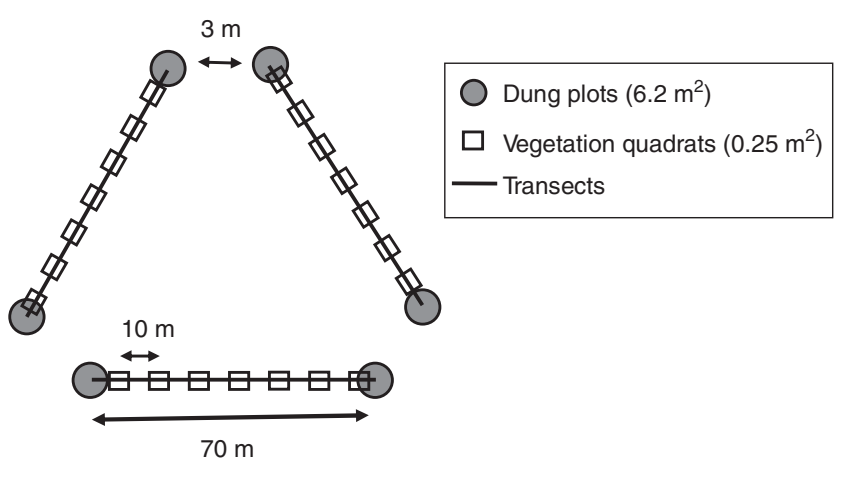

Fig. 2. Layout of 21 vegetation quadrats and six dung plots at a site (not to scale). Dung plots were a minimum of $3 \mathrm{~m}$ apart.

to September 2004, coinciding with the monitoring of the density of kangaroos.

\section{Data analysis}

A cumulative sum of deviations (CUSUM) was calculated using the long-term mean for rainfall in Idalia National Park between 1990 and 2004 to determine how dry our study period was relative to other periods, following Kerle et al. (2007). Kangaroo density for each sample time was estimated following the analysis guidelines of Buckland et al. (1993) and by using the associated program Distance 3.5 (Burnham et al. 1980). The sighting objects were clusters (or groups) of kangaroos. The counts from Treatment Area 1 and the control areas were low (i.e. $<40$ ) and, therefore, were pooled to model detection probability. Detection probability was modelled with a uniform key function, with several cosine adjustments determined by Akaike's information criterion. Clusters more than $250 \mathrm{~m}$ from the transects were disregarded because outlying observation is difficult to model and contributes little to the density estimation (Buckland et al. 1993; Southwell and Weaver 1993). All statistical analyses were performed with R 2.7.0. (R Development Core Team 2008). All data were logtransformed to meet the assumptions of normality (Zar 1999). The effect of fencing waterpoints on the monthly changes in the density of kangaroos was measured because densities varied between the four areas. We added a small value (0.01) to each of the estimated densities before log-transformation because some densities were zero. The change in the density of kangaroos for each period in each of the four areas was calculated by subtracting the log-estimated density of kangaroos from the log-estimated density in the previous sample time. The change was then divided by the number of months since the last monitoring to calculate the log monthly change in the density of kangaroos for each period. The effect of waterpoint status (fenced or unfenced) on the log monthly change in the density of kangaroos was examined with a GLM (Crawley 2002). Control WP1 became dry from June 2002 to January 2003 owing to drought, thus failing to act as an experimental control. We omitted data gathered during this period from this area from all the statistical analyses.

The mean number of dung pellets per square metre was calculated for each distance (by first averaging the six plots, and then the three sites at each distance). We divided this figure by the number of months since the last dung count, to calculate the mean monthly rates of dung pellets deposited. Hereafter, this will be referred to as kangaroo 'grazing pressure'. The higher the figure, the more time kangaroos were thought to have spent feeding there. Because a kangaroo's reliance on artificial waterpoints depends on seasons (e.g. Montague-Drake and Croft 2004), a linear mixed effect model (Crawley 2002) was used to determine the magnitude of the changes in the spatial distribution of grazing pressure from dry to wet seasons in the areas with fenced waterpoints relative to those with unfenced waterpoints. From the park's rainfall data, we specified the following periods to be dry seasons: June 2002-October 2002, March 2003, June 2003, June 2003-September 2003 and April 2004-September 2004. The remaining study periods were classified as wet seasons. Season, waterpoint status (fenced or unfenced), distance from the waterpoint and their interactions were specified as fixed effects. Areas were specified as random effects. A significant interaction among season, waterpoint status and distance would indicate that the magnitude of the difference from dry to wet seasons changed in the areas with fenced waterpoints relative to the areas with unfenced waterpoints. For this analysis, we omitted data from the periods during which waterpoints were being fenced off. When a significant interaction between waterpoint status and distance was found, the effect of distance on grazing pressure was determined with a separate GLM for fenced and unfenced waterpoints.

To determine the relationship between the grazing pressure and the density of kangaroos, the grazing pressure for each of the four areas for each period was calculated by averaging over the distances to yield the grazing pressure per area to match the density of kangaroos, which was estimated for each area rather than for each distance. Because the density of kangaroos was estimated for a point in time rather than a period, the density between two sequential times (e.g. June 2002 and October 2002) was averaged to match the eight estimates of grazing pressure. The relationship between the kangaroo grazing pressure and density of kangaroos was assessed with a Spearman rank correlation test. Unlike other analyses, no data were omitted for this test.

We used a graphical analysis based on the Lotka-Volterra predator-prey model (May 1973) to examine the relationship between vegetation biomass and grazing pressure. The Lotka-Volterra model describes the changes over time in the size of interacting predator and prey populations. Herbivore consumption influences the rate of renewal of food plants. This, in turn, influences the dynamics of the herbivore population itself (Caughley and Lawton 1981). If the herbivore population is limited by food abundance, the time sequence of this cycle - when presented graphically - will show a negativefeedback loop generated by the influence that herbivores and vegetation exert over one another's abundance (Bayliss and Choquenot 2002). We used the biomass of perennial grasses because long-term exclosures at the park revealed a substantial decrease in these plants owing to kangaroo grazing (Fukuda 2006), a fact in accord with the findings of previous studies (Norbury and Norbury 1992; Norbury et al. 1993). Because the biomass of perennial grasses and grazing pressure varied within the four areas, we investigated plant-herbivore dynamics in each area separately. The mean grazing pressure between two 
sequential periods was calculated for each area because we wanted to compare the grazing pressure and the biomass of perennial grasses at a given time (cf. grazing pressure was measured as monthly rates of dung pellets deposited over a period - see earlier section). After averaging, we gained seven data points per area for grazing pressure. The percentage cover and height of perennial grasses within a quadrat were multiplied to produce a biomass index. The mean biomass index of perennial grasses was calculated for each area (by first averaging 21 quadrats, then the three sites at each distance and finally the five distances in each area). In contrast to grazing pressure, vegetation biomass was monitored nine times during the study period. To yield sample sizes equal (i.e. seven) to those for the grazing pressure, the area mean of biomass indices for perennial grasses between two sequential sample times was calculated (e.g. $i$ : June 2002 and October 2002; and ii: October 2002 and January 2003) and these were then averaged again (i.e. averaged over $i$ and $i i)$. Averaging was used in the analysis to remove both spatial and temporal autocorrelation. For each area, a corresponding pair of log-transformed biomass indices and a grazing pressure figure per sampling time were plotted against each other. We then visually analysed how this relationship changed over time.

\section{Results}

The 12-month period starting from August 2002 (2 months into the study period) was the driest 12-month-period since the park was gazetted in 1990, according to the CUSUM analysis (Fig. 3). The rest of the study period was also drier than average. The density of kangaroos in areas surrounding a fenced waterpoint declined whereas the density in areas surrounding an unfenced waterpoint increased in the period immediately after fencing (Fig. 4). Subsequently, however, the monthly changes in the density around fenced and unfenced waterpoints remained similar for the rest of the study period. This apparent lack of difference

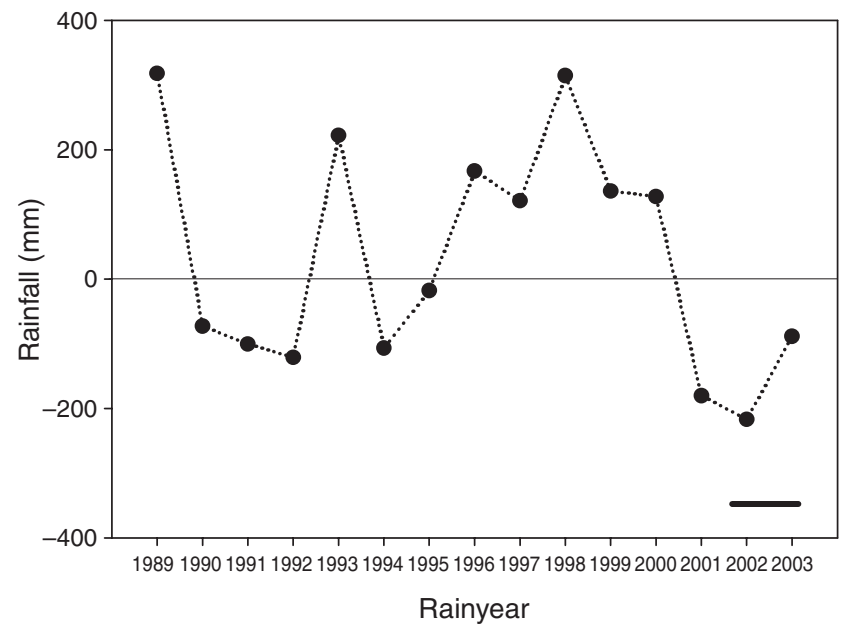

Fig. 3. Rainfall in Idalia National Park from 1989 to 2003 displayed as a cumulative sum of deviations from the long-term mean rainfall (CUSUM). Because a rain-year starts in August, rain-year 2002 refers to the period from August 2002 to July 2003. The horizontal line at $0 \mathrm{~mm}$ indicates the 'average' rain-year. The thick horizontal line at the bottom indicates our study period. was supported by non-significant differences in the $\log$ mean monthly change in the density of kangaroos between the areas with unfenced waterpoints $(0.07 \pm 0.11$ individuals per square kilometre, mean \pm 1 s.e.) and the areas with fenced waterpoints $\left(0.03 \pm 0.10, t_{26}=-0.25, P=0.8\right)$. This indicates that fencing off waterpoints had no lasting influence on kangaroos, even if it disturbed their distribution in the short-term.

Similarly, our prediction that the kangaroo grazing pressure would become more focussed around unfenced waterpoints, particularly during dry seasons, was not supported by the data, because none of the interaction terms involving season proved significant $(P>0.7)$. The kangaroo grazing pressure was not focussed around unfenced waterpoints, irrespective of seasons. Even though the interaction between the waterpoint status and the distance was significant (parameter $=-0.0112$, s.e. $=0.00309$, $t_{125}=3.64, \quad P<0.0005$ ), the grazing pressure showed no significant trend in regard to the distance from the waterpoints in areas with unfenced waterpoints (Fig. 5, $t_{73}=1.33, P=0.2$ ), whereas in areas with fenced waterpoints, the grazing pressure was higher at sites closer to the fenced waterpoints $\left(t_{56}=-2.40\right.$, $P=0.02$ ). Furthermore, the grazing pressure at sites 0.5 and $1 \mathrm{~km}$ from the fenced waterpoints was higher than that at identical distances from unfenced waterpoints (Fig. 5).

The graphical analysis of the time sequence of plant-herbivore dynamics suggested that the biomass indices of perennial grasses and the kangaroo grazing pressure interacted closely over time, showing negative-feedback loops in all four areas (Fig. 6). As the drought intensified in October 2002, the kangaroo grazing pressure started to decline. This reduction led to an increase in grass biomass. The recovery of the kangaroo population in response to this increased food abundance, following the drought, was slow and lagged. The grazing pressure showed only a slight increase in June 2003, after an 8-month period of

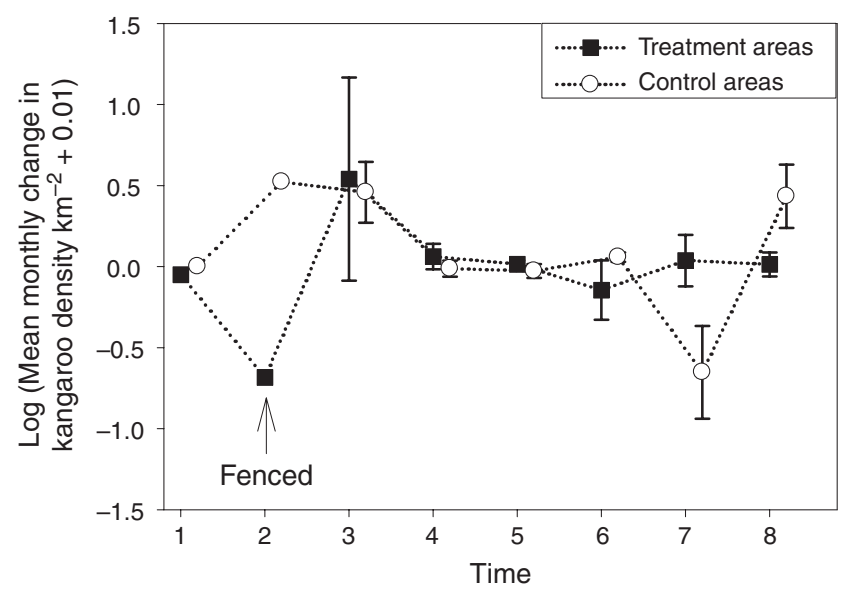

Fig. 4. The logged mean ( \pm 1 s.e. $)$ monthly change in the density of kangaroos (per square kilometre) in control and treatment areas. The arrow indicates when the fencing off both treatment waterpoints was completed. We omitted data from the periods during which waterpoints were being fenced off and when Control WP1 lost its water: $n=1$ for Time 1 and 2; and $n=2$ for the rest of the study period. Time $1=$ June-October 2002, Time $2=$ October 2002-January 2003, Time 3 = January-March 2003, Time $4=$ March-June 2003, Time $5=$ June-September 2003, Time $6=$ September 2003-January 2004, Time 7 = January-April 2004 and Time 8=April-September 2004. 


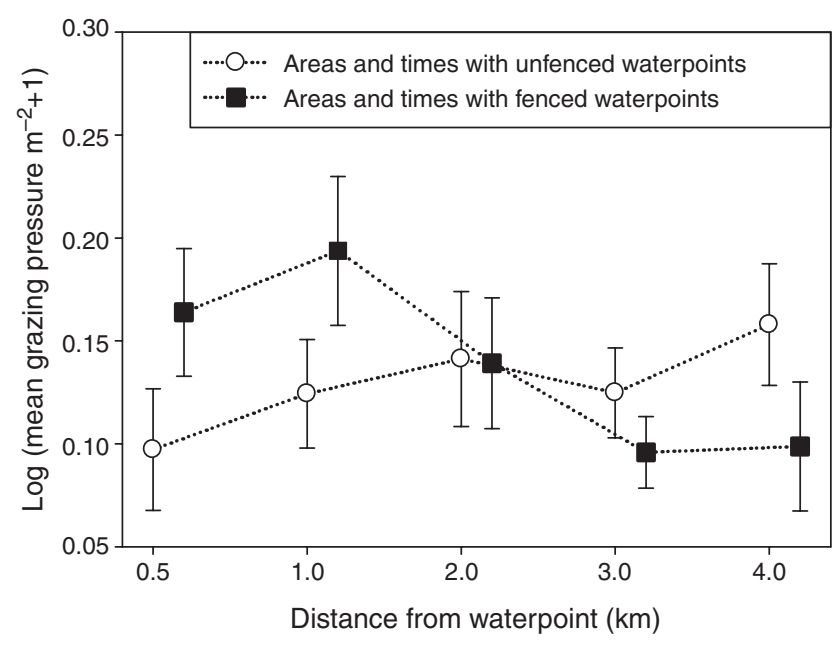

Fig. 5. The logged mean $( \pm 1$ s.e. $)$ grazing pressure over five distances from waterpoints in areas and times with unfenced waterpoints $(n=15$ for each distance) and those with fenced waterpoints ( $n=13$ for distances between 0.5 and $3 \mathrm{~km}$, and $n=6$ for $4 \mathrm{~km}$ ). The former areas include data from treatment areas before fencing. The grazing pressure was measured as a monthly rate of dung pellets deposited per square metre. increased grass biomass. The increased grazing pressure reduced grass biomass, which in turn limited and reduced the grazing pressure in September 2003. The grazing pressure continued to decline until January 2004, when the cycle was completed. In April 2004, there was a slight recovery in the grazing pressure. However, the effect of the increased food abundance on the grazing pressure appears to be lagged, similar to that immediately following the drought. The population recovery was slow; the grazing pressure remained at the same level or continued to decline for another 10 months, starting from June 2003, in spite of the increased biomass of perennial grasses following the drought (Fig. 6). A positive correlation was found between the density of kangaroos and the grazing pressure $(r=0.531$, d.f. $=31, P<0.001)$.

\section{Discussion}

Our results provided convincing evidence that fencing off artificial waterpoints does not influence the density of kangaroos over a distance of up to $4 \mathrm{~km}$ when these conditions are maintained over a period of up to 25 months, even when the climatic conditions are dry. Previous studies were terminated within 4 months since the waterpoints were removed (a) Treatment Area 1

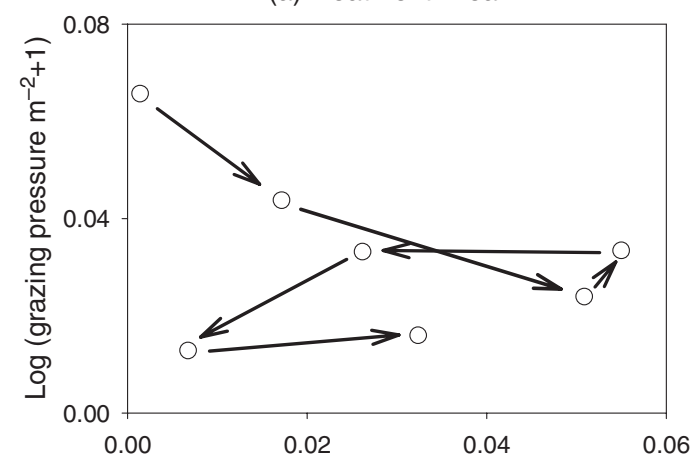

(c) Control Area 1

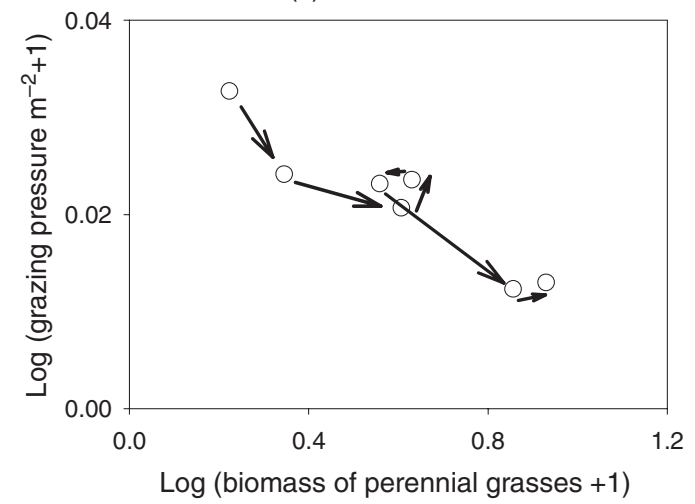

(b) Treatment Area 2

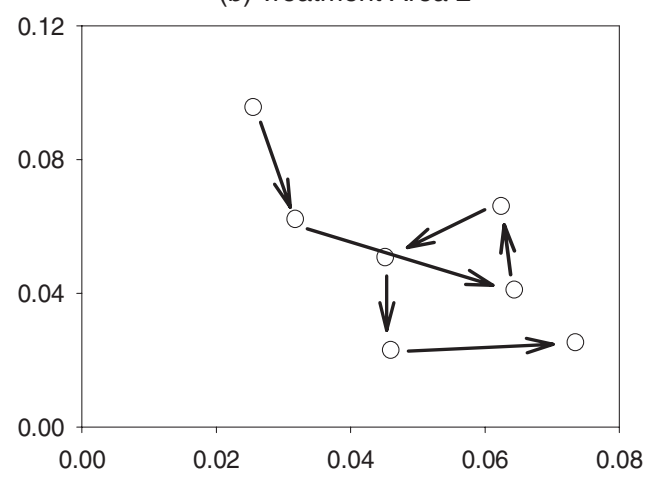

(d) Control Area 2

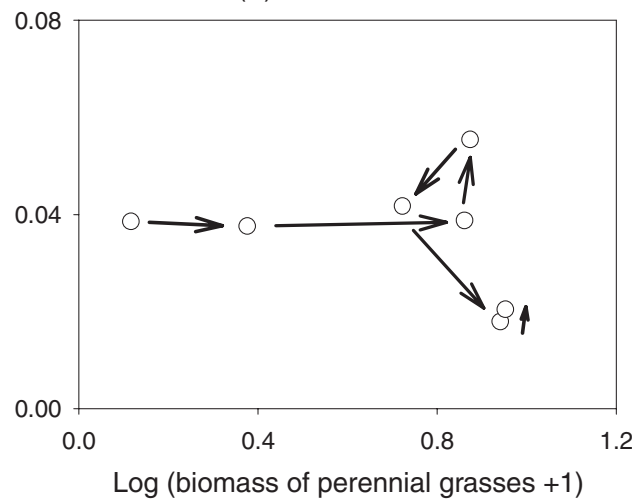

Fig. 6. The relationship between the kangaroo grazing pressure and the biomass of perennial grasses in (a) Treatment Area 1, (b) Treatment Area 2, (c) Control Area 1 and (d) Control Area 2 in Idalia National Park. The arrows indicate the time sequence in the relationship of October 2002, January 2003, March 2003, June 2003, September 2003, January 2004 and April 2004. The data were log-transformed and scales in each area were adjusted so that any negative-feedback pattern in each area could be identified. The grazing pressure was measured as the number of dung pellets deposited per square metre. 
(Gibson 1995; Montague-Drake 2004) and/or waterpoint removal was followed by conditions of above-average precipitation which reduced the effects on herbivore density and distribution (Montague-Drake 2004; Cain et al. 2008). Therefore, one of the main strengths of our study was that the waterpoint removal coincided with a severe drought and the conditions that followed were also dry.

Our results also showed that kangaroos forage irrespective of the proximity of unfenced waterpoints. If water were an influential factor in determining the spatial distribution of the kangaroo grazing pressure, we would expect a significant interaction between the status and distance of the waterpoint, i.e. the grazing pressure should be higher at sites closer to unfenced waterpoints, whereas the grazing pressure in areas with fenced waterpoints should be distributed irrespective of the distance from the waterpoints. Although the interaction was significant, the pattern was opposite to the one we predicted. Underhill et al. (2007) assessed the physiological needs of kangaroos at the park and concluded that, regardless of the body size, they all would have relied on waterpoints for drinking by July 2002 (a month after the commencement of our study) because water from forage would have been insufficient to provide their needs. There was no evidence that kangaroos switched their diets to satisfy water requirements from forage following the fencing off the waterpoints (Fukuda 2006). These, along with the dry conditions of the rest of our study period, imply that kangaroos visited alternative waterpoints but returned to the treatment areas to forage. Consequently, there was no change in the density or distribution following fencing off the treatment waterpoints, even during dry seasons. Our results support the conclusion of Freudenberger and Hacker (1995), Gibson (1995) and Montague-Drake (2004) that removal of kangaroos' access to water from one area will not reduce the total density or grazing pressure. Our results are also in accord with the conclusion reached by Cain et al. (2008) who experimentally removed permanent water sources and found that there was no impact on the survival rates and home ranges of desert bighorn sheep (Ovis Canadensis mexicana) in Arizona, USA. Farmers on properties surrounding conservation areas are raising concerns about whether the removal of waterpoints in reserves leads to kangaroos being forced to drink at off-park locations. Our results suggest, however, that even if kangaroos did drink from waterpoints at off-park locations immediately after the removal of waterpoints in conservation areas, the effect would be only temporary. In the longer term, it is unlikely to result in a shift in grazing distribution. Nevertheless, telemetry studies before and after experimental removal of waterpoints need to be conducted to further ascertain this.

Our study detected a link between the kangaroo grazing pressure and food abundance, providing empirical support for the Lotka-Volterra predator-prey model. The sign of population regulation (i.e. a negative-feedback loop) suggests that food abundance, rather than availability of unfenced waterpoints within a range of $4 \mathrm{~km}$, determines the kangaroo grazing pressure. The initial decline in the grazing pressure followed by the delayed increase, in spite of a more rapid recovery in food abundance, was similar to the finding by Bayliss and Choquenot (2002) from their time-trace graphical analysis on the rate of increase in red kangaroos against pasture biomass. The negative- feedback loops indicate that the kangaroo population is regulated extrinsically by food abundance. Although other herbivores were also present in the park, the average density of wallaroos (usually the most common species of large herbivores) from our walked counts was only 0.51 individuals per square kilometre (Fukuda 2006). Pellets of other herbivores were also rarely detected in our dung-pellet counting plots. We, therefore, conclude that the major mammalian herbivore in our study site is the red kangaroo. The close link is consistent with earlier work, showing that the distribution of the kangaroo in central Australia and in northwestern New South Wales is determined primarily by food abundance, and not by the location of waterpoints (Newsome 1965; Landsberg and Stol 1996). Our results are also in agreement with the finding of Cain et al. (2008) for desert bighorn sheep that food quantity and quality are the primary limiting factors, rather than availability of a permanent water source. The positive correlation between the density of kangaroos and the grazing pressure supports the use of dung counts to describe the grazing pressure.

The close link between food abundance and the kangaroo grazing pressure illustrates the fundamental difficulty in reducing the kangaroo grazing pressure and regenerating vegetation in many parts of the rangelands, because very few areas are beyond the kangaroos' convenient reach. Vegetation regeneration may occur only if the area is sufficiently remote from permanent waterpoints (Landsberg et al. 2003) and fencing will become effective only when access is prohibited to all waterpoints within a close range of the kangaroo's home range. Fensham and Fairfax (2008) recently proposed $7 \mathrm{~km}$ to be the threshold distance for red kangaroos. Unfortunately, our dataset has little to contribute to this larger discussion because we examined the effects of fencing of waterpoints only over 4-km distance. Accumulating evidence from distribution studies suggests that some rarer plants and animals are being displaced by other species that are more favoured around artificial waterpoints in Australia (Landsberg et al. 2003) and Africa (de Leeuw et al. 2001; Smit et al. 2007). In parks created within Australian rangelands where there has previously been livestock grazing around waterpoints, separating historical grazing impact from current effects caused by kangaroos remains a challenging issue (Montague-Drake and Croft 2004; Fukuda 2006). Although it may be tempting to install haphazard fencing, constraining access to water by wildlife requires in-depth planning. Further removal of waterpoints needs to be accompanied with experimental work testing carefully formulated hypotheses, because the effects on the flora and fauna (especially threatened species) of fencing off artificial waterpoints within the national parks are not easily predictable. A useful approach would be to remove isolated artificial waterpoints first, so that the distances between the remaining waterpoints are maximised, as suggested by Thrash and Derry (1999). To accommodate water requirements of species of particular interest, e.g. threatened species such as bridled nailtail wallabies in Idalia National Park, water may have to be provided by using a trough that allows selective access (e.g. by placing the trough behind a kangaroo-proof fence). Where waterpoints are left in place for kangaroos and other wildlife to use, the regular removal of accumulating mud is necessary to prevent animals becoming trapped as water levels recede during droughts (Montague-Drake 2004; Fukuda 2006). 
Although we found no effect on red kangaroos, the effect of fencing off waterpoints may be much greater on common wallaroos, which have a more limited mobility compared with red kangaroos (see Ealey 1967), at least during dry conditions when forage and ephemeral sources provide insufficient water. For example, wallaroos have an average annual home range of only $1-3 \mathrm{~km}^{-2}$ in arid regions (Clancy and Croft 1990). It is worth noting that recolonisation of the study area by wallaroos was much slower than that of red kangaroos, and that the fencing off the two waterpoints, located near the park border, might have prevented recolonisation altogether (Fukuda 2006). In future studies, greater replication would be desirable, as well as more before-fencing data (e.g. at least one full seasonal cycle) from both treatment and control areas, particularly from areas with high density of $M$. robustus.

\section{Acknowledgements}

We express our gratitude to the park rangers, C. and M. Morgan, and to the many volunteers who assisted with field work. We thank M. Laidlaw for her help on our study map. We also thank G. Lundie-Jenkins for providing information on kangaroo density estimates and the QPWS for providing field accommodation. We are grateful to Drs K. McConkey, G. Norbury and A. Smyth, and three anonymous reviewers for their useful comments on our manuscript. We acknowledge financial support from an ARC Linkage Grant to H. Possingham, G. Grigg and S. Phinn and also from the School of Integrative Biology, the University of Queensland.

\section{References}

Bayliss, P. (1987). Kangaroo dynamics. In 'Kangaroos: Their Ecology and Management in the Sheep Rangelands of Australia'. (Eds G. Caughley, N. Shepherd and J. Short.) pp. 119-134. (Cambridge University Press: Cambridge, UK.)

Bayliss, P., and Choquenot, D. (2002). The numerical response: rate of increase and food limitation in herbivores and predators. Philosophical Transactions of the Royal Society of London Series B 357, 1233-1248. doi: 10.1098/rstb.2002.1124

Bennett, B. (1997). Water points: where pastoralism and biodiversity meet. Ecos 92, 10-14.

Buckland, S. T., Anderson, D. R., Burnham, B. K., and Laake, J. L. (1993). 'Distance Sampling: Estimating Abundance of Biological Populations.' (Chapman and Hall: London.)

Burnham, K. P., Anderson, D. R., and Laake, J. L. (1980). Estimation of density from line transect sampling of biological populations. Wildlife Monographs 72, 1-202.

Cain, J. W., Krausman, P. R., Morgart, J. R., Jansen, B. D., and Pepper, M. P. (2008). Responses of desert bighorn sheep to removal of water sources. Wildife Monographs 171, 1-30. doi: 10.2193/2007-209

Calaby, J. H., and Grigg, G. C. (1989). Changes in macropodoid communities and populations in the past 200 years, and the future. In 'Kangaroos, Wallabies and Rat-kangaroos, Vol. 2'. (Eds G. C. Grigg, P. Jarman and I. Hume.) pp. 813-820. (Surrey Beatty: Sydney.)

Caughley, G., and Lawton, J. H. (1981). Plant-herbivore systems. In 'Theoretical Ecology'. (Ed. R. M. May.) pp. 132-166. (Blackwell: Oxford.)

Clancy, T. F., and Croft, D. B. (1990). Home range of the common wallaroo, Macropus robustus erubescens, in far western New South Wales. Australian Wildlife Research 17, 659-673. doi: 10.1071/ WR9900659

Crawley, M. J. (2002). 'Statistical Computing: An Introduction to Data Analysis Using S-Plus.' (John Wiley \& Sons Ltd: Oxford, UK.) de Leeuw, J., Waweru, M. N., Okello, O. O., Maloba, M., Nguru, P., Said, M. Y., Aligula, H. M., Heitkonig, I. M. A., and Reid, R. S. (2001). Distribution and diversity of wildlife in northern Kenya in relation to livestock and permanent water points. Biological Conservation 100, 297-306. doi: 10.1016/S0006-3207(01)00034-9

Department of Environment (1998). Idalia National Park: draft management plan. Department of Environment, Brisbane.

Ealey, E. H. M. (1967). Ecology of the euro, Macropus robustus (Gould), in north-western Australia. II. Behaviour, movements and drinking patterns. CSIRO Wildlife Research 12, 27-51.

Fensham, R. J., and Fairfax, R. J. (2008). Water-remoteness for grazing relief in Australian arid-lands. Biological Conservation 141, 1447-1460. doi: 10.1016/j.biocon.2008.03.016

Freudenberger, D., and Hacker, R. B. (1995). The short-term effect of selectively excluding kangaroos from stock watering points. In 'Proceedings 10th Australian Vertebrate Pest Control Conference, Hobart, Tasmania, 29 May-2 June 1995'. pp. 309-312. (Department of Primary Industry and Fisheries: Hobart.)

Fukuda, Y. (2006). The effects of closing artificial watering points on recolonisation by large macropods and landscape rehabilitation in a semi-arid national park. Ph.D. Thesis, The University of Queensland, Brisbane.

Gibson, L. A. (1995). Concentration of eastern grey kangaroos (Macropus giganteus), red kangaroos (Macropus rufus) and wallaroos (Macropus robustus) in the vicinity of artificial waters and differences in artificial water usage. In 'Ecological Research and Management in the Mulgalands'. (Eds M. Page and T. S. Beutel.) pp. 75-84. (The University of Queensland: Gatton, Qld.)

Grice, A. C., and Barchia, I. (1992). Does grazing reduce survival of indigenous perennial grasses of the semi-arid woodlands of western New South Wales? Australian Journal of Ecology 17, 195-205. doi: 10.1111/j.1442-9993.1992.tb00798.x

Hacker, R. B., and Freudenberger, D. (1997). The effect of short-term exclosure of watering points on the behaviour and harvesting efficiency of grey and red kangaroos. Rangeland Journal 19, 145-156. doi: 10.1071/RJ9970145

Harrington, R., Owen-Smith, N., Viljoen, P. C., Biggs, H. C., Mason, D. R., and Funston, P. (1999). Establishing the causes of the roan antelope decline in the Kruger National Park, South Africa. Biological Conservation 90, 69-78. doi: 10.1016/S0006-3207(98)00120-7

James, C. D., Landsberg, J., and Morton, S. R. (1999). Provision of watering points in the Australian arid zone: a review of effects on biota. Journal of Arid Environments 41, 87-121. doi: 10.1006/jare.1998.0467

Kerle, J. A., Fleming, M. R., and Foukles, J. N. (2007). Managing biodiversity in arid Australia: a landscape view. In 'Animals of Arid Australia: Out on Their Own?' (Eds C. Dickman, D. Lunney and S. Bergin.) pp. 42-64. (Royal Zoological Society of New South Wales: Sydney.)

Landsberg, J., and Stol, J. (1996). Spatial distribution of sheep, feral goats and kangaroos in woody rangeland paddocks. Rangeland Journal 18, 270-291. doi: 10.1071/RJ9960270

Landsberg, J., James, C. D., Morton, S. R., Hobbs, T., Stol, J., Drew, A., and Tongway, H. (1997). 'The Effects of Artificial Sources of Water on Rangeland Biodiversity.' (Environmental Australia and CSIRO: Canberra.)

Landsberg, J., James, C. D., Morton, S. R., Muller, W. J., and Stol, J. (2003). Abundance and composition of plant species along grazing gradients in Australian rangelands. Journal of Applied Ecology 40, 1008-1024. doi: 10.1111/j.1365-2664.2003.00862.x

May, R. M. (1973). 'Stability and Complexity in Model Ecosystems.' (Princeton University Press: Princeton, NJ.)

McCarron, H. C. K., and Dawson, T. J. (1989). Thermal relations of Macropodoidea in hot environments. In 'Kangaroos, Wallabies and Rat-kangaroos, Vol. 1'. (Eds G. Grigg, P. Jarman and I. Hume.) pp. 255-263. (Surrey Beatty: Sydney.) 
McCullough, D. R., and McCullough, Y. (2000). 'Kangaroos in Outback Australia.' (Columbia University Press: New York.)

Montague-Drake, R. (2004). Strategic management of artificial watering points for biodiversity conservation. Ph.D. Thesis, The University of New South Wales, Sydney.

Montague-Drake, R., and Croft, D. B. (2004). Do kangaroos exhibit waterfocused grazing patterns in arid New South Wales? A case study in Sturt National Park. Australian Mammalogy 26, 87-100.

Newsome, A. E. (1965). The distribution of red kangaroos, Megaleia rufa (Desmarest), about sources of persistent food and water in central Australia. Australian Journal of Zoology 13, 289-299. doi: 10.1071/ ZO9650289

Norbury, G. (1992). An electrified watering trough that selectively excludes kangaroos. Rangeland Journal 14, 3-8. doi: 10.1071/RJ9920003

Norbury, G., and Norbury, D. (1992). The impact of red kangaroos on rangeland regeneration. In 'Proceedings of the 7th Australian Rangeland Society Biennial Conference: Australian Rangelands in a Changing Environment, Cobar, NSW, 5-8 October 1992'. pp. 344-347. (Australian Rangeland Society.)

Norbury, G. L., Norbury, D. C., and Hacker, R. B. (1993). Impact of red kangaroos on the pasture layer in the Western Australian arid zone. Rangeland Journal 15, 12-23. doi: 10.1071/RJ9930012

Norbury, G. L., Norbury, D. C., and Oliver, A. J. (1994). Facultative behaviour in unpredictable environments: mobility of red kangaroos in arid Western Australia. Journal of Animal Ecology 63, 410-418. doi: 10.2307/5558

Owen-Smith, N. (1996). Ecological guidelines for waterpoints in extensive protected areas. South African Wildlife Research 26, 107-112.

Page, M. (1997). Vegetation dynamics in the Mulgalands: a Currawinya National Park case study. Ph.D. Thesis, The University of Queensland, Brisbane.

Pople, A. R. (2006). Modelling the spatial and temporal dynamics of kangaroo populations for harvest management. Department of Environment and Heritage, Canberra.

Pople, A. R., and McLeod, S. (2000). Kangaroo management and the sustainable use of rangelands. In 'Management for Sustainable Ecosystems'. (Eds P. Hale, A. Petrie, D. Moloney and P. Sattler.) pp. 78-86. (Centre for Conservation Biology, The University of Queensland: Brisbane.)

Pople, A. R., and Page, M. (2001). Management of artificial water points on national parks in western Queensland. Queensland Parks and Wildlife Service, Brisbane.

Pople, A. R., Lowry, J., Lundie-Jenkins, G., Clancy, T. F., McCallum, H. I., Sigg, D., Hoolihan, D., and Hamilton, S. (2001). Demography of bridled nailtail wallabies translocated to the edge of their former range from captive and wild stock. Biological Conservation 102, 285-299. doi: 10.1016/S0006-3207(01)00101-X
R Development Core Team (2008). 'R 2.7.0.' (R Foundation for Statistical Computing: Vienna, Austria.)

Rosenstock, S. S., Ballard, W. B., and Devos, J. C. (1999). Viewpoint: benefits and impacts of wildlife water developments. Journal of Range Management 52, 302-311. doi: 10.2307/4003538

Scanlan, J. C. (2002). Some aspects of tree-grass dynamics in Queensland's grazing lands. Rangeland Journal 24, 56-82. doi: 10.1071/RJ02003

Sluiter, I. R. K., Allen, G. G., Morgan, D. G., and Walker, I. S. (1997). Vegetation responses to stratified kangaroo grazing pressure at Hattah-Kulkyne National Park, 1992-96. Department of Natural Resources and Environment, Melbourne.

Smit, I. P. J., Grant, C. C., and Devereux, B. J. (2007). Do artificial waterholes influence the way herbivores use the landscape? Herbivore distribution patterns around rivers and artificial surface water sources in a large African savanna park. Biological Conservation 136, 85-99. doi: 10.1016/ j.biocon.2006.11.009

Southwell, C. (1994). Evaluation of walked line transect counts for estimating macropod density. Journal of Wildlife Management 58, 348-356. doi: $10.2307 / 3809401$

Southwell, C., and Weaver, K. (1993). Evaluation of analytical procedures for density-estimation from line-transect data - data grouping, data truncation and the unit of analysis. Wildlife Research 20, 433-444. doi: 10.1071/ WR9930433

Thrash, I., and Derry, J. F. (1999). The nature and modelling of piospheres: a review. Koedoe 42, 73-94.

Triggs, B. (1984). 'Mammal Tracks and Signs: A Field Guide for Southeastern Australia.' (Oxford University Press: Melbourne.)

Underhill, S., Grigg, G. C., Pople, A. R., and Yates, D. J. (2007). A physiological assessment of the use of water point closures to control kangaroo numbers. Wildlife Research 34, 280-287.

Western, D. (1975). Water availability and its influence on the structure and dynamics of a savannah large mammal community. East African Wildlife Journal 13, 265-286.

Zar, J. H. (1999). 'Biostatistical Analysis.' 4th edn. (Prentice-Hall: Upper Saddle River, NJ.)

Manuscript received 2 September 2008, accepted 22 May 2009 